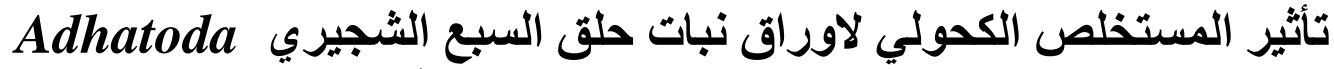 vasica Ceratitis capitata
}

\author{
هبه شاكر أحدد*
}

\author{
أرياد /حمد الطويل*** \\ استلام البحث 3، تشرين الاول، 270 2010 2010

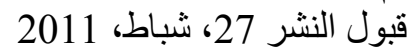

عماد أحمد محمود*

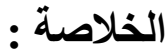

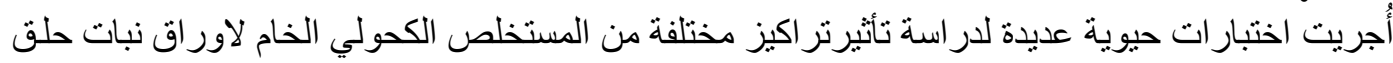

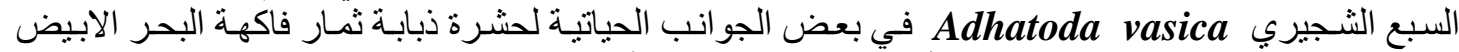

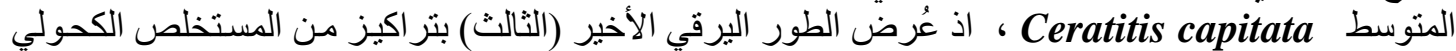

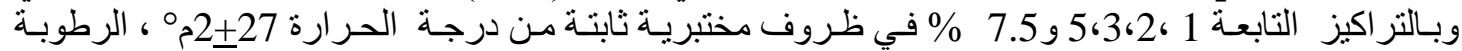

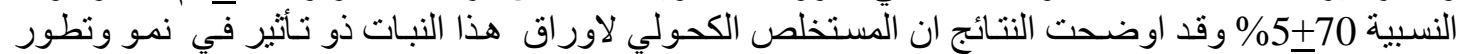

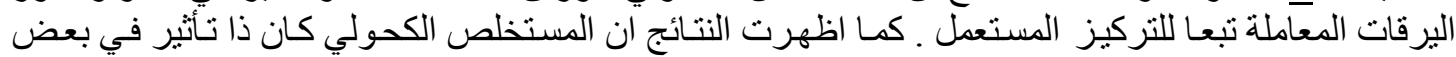

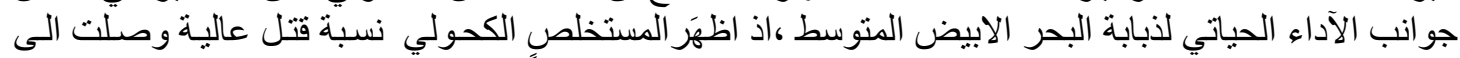

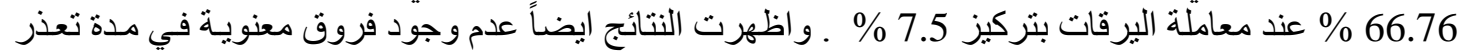

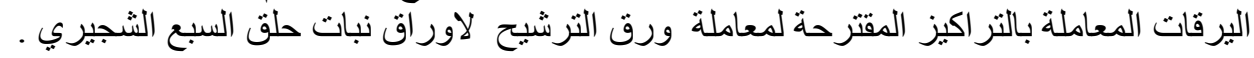

الكلمات المفتاحية :مستخلص اوراق نبات حلق السبع الشجيري ، ذبابة البحر الابيض المتوسط
\end{abstract}

C كما ان الاور اق غنيـة بفيتـامين Oxyvasicine

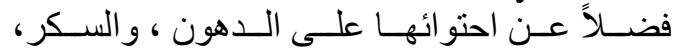

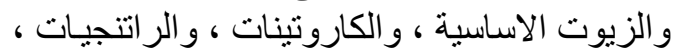

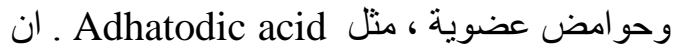

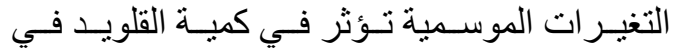

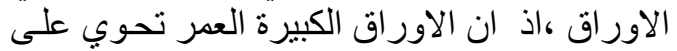

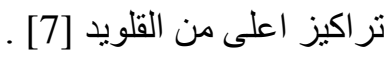

$$
\text { جمع العينات :- الموائق العمل : }
$$

تم الحصول على عينات ذبابة البحر الابيض

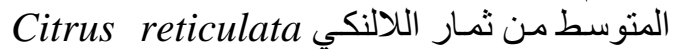

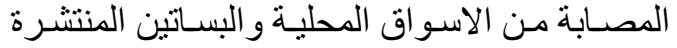

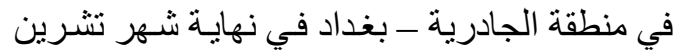
الثاني وكانون الاول من عام 2007 ـ اما المشمش فئش Prunus armeniaca

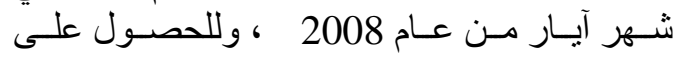

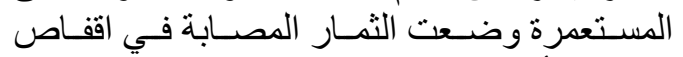

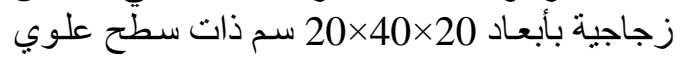

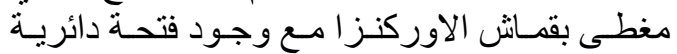
بقطر 14سم في احدى جهات الققص محاطة بقدماش دأش الاوركنزا المثبت بشكل اسطو اني منطساول رُبطت المبت

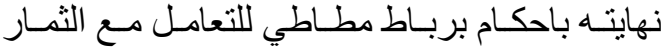

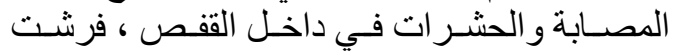

المقدمة :

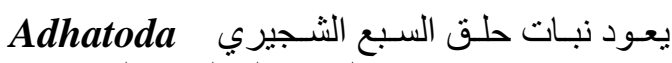
vasica_ness

Acanthaceae

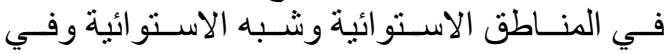

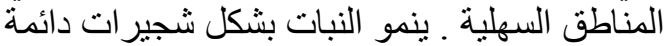
الخضرة, والاور اق عريضة ورئة ورمية الثنكل طويلة

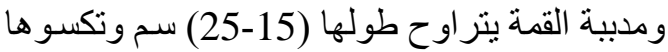

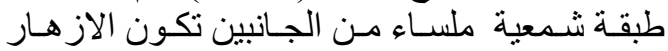

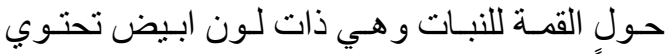

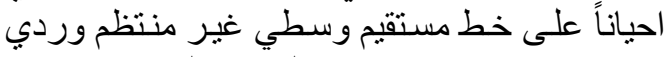

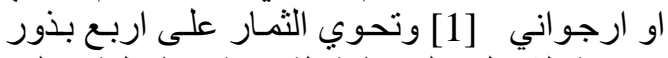

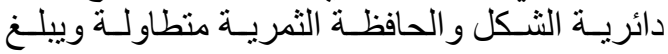

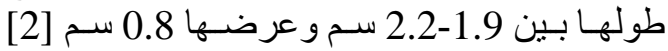

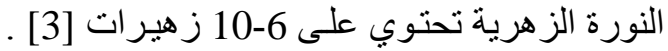

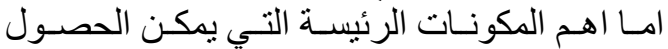

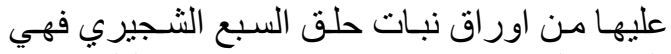

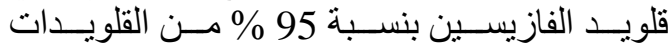
المعزولة وتركيبه الكيميائي

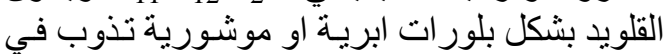

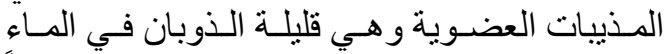

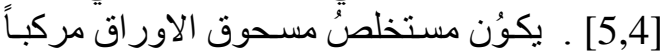

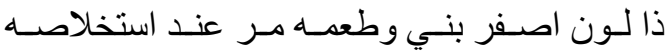

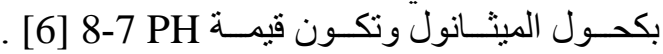

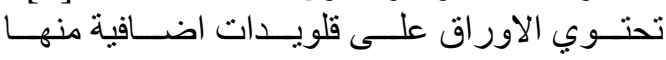
Deoxyvasicine, Maiontone

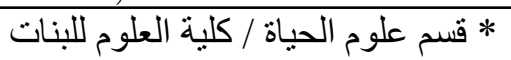
**مركز البحوث الزر اعية/وزارة العلوم و التكنلوجيا 
الاور اق الفتيــة غيــر المصــابة بــامر اض نباتيــــة

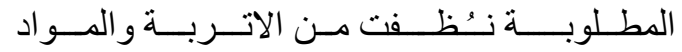

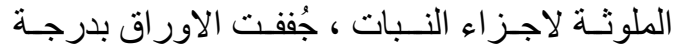

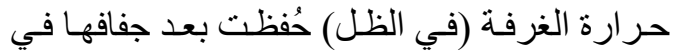

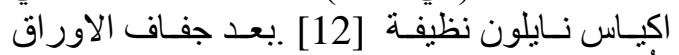

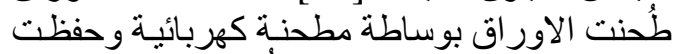

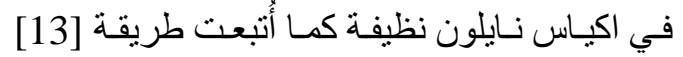
للحصول على المستخلص الكحولي لاور اق نبات التهات

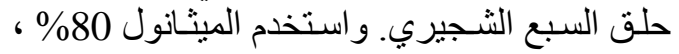

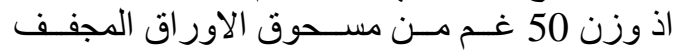
ووضـع المسحوق في Thimble ونقل الــ الى جهاز Soxhlet

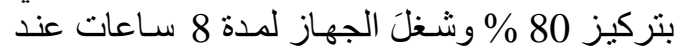

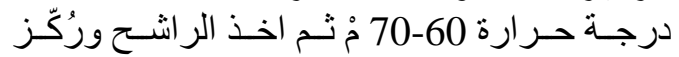

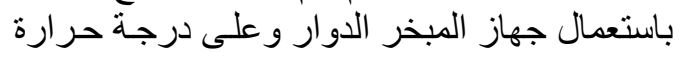

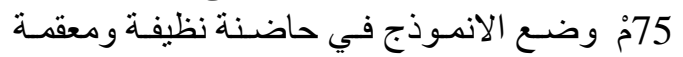

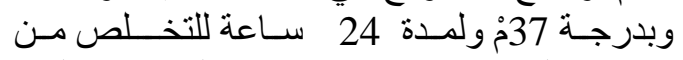

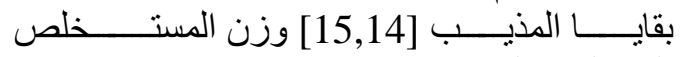

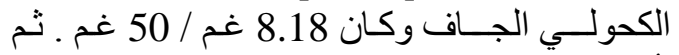
عُوملت برقات الطور الثالث (الاخير) لذبابة البحر التبر

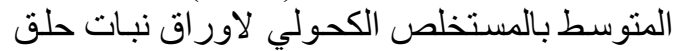

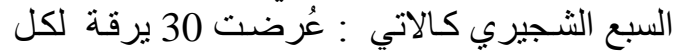

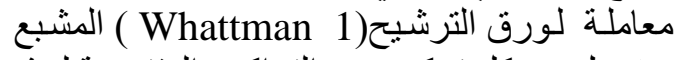
ب5 مل من كل تركيز من التر اكيز المقترحة لهذه

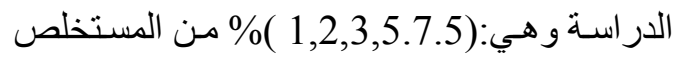

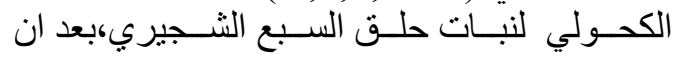

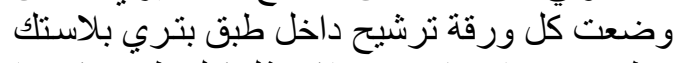

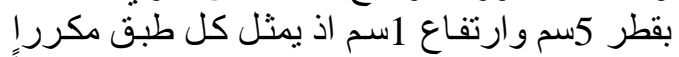

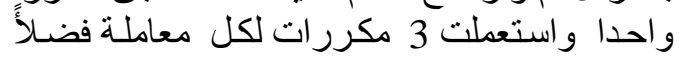

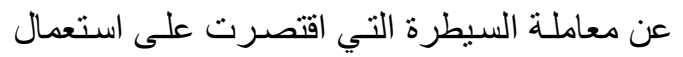

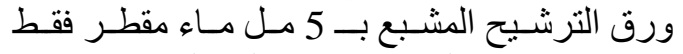

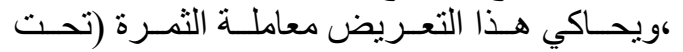

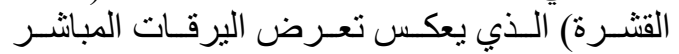

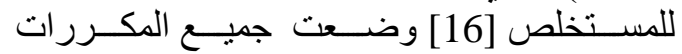

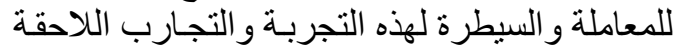

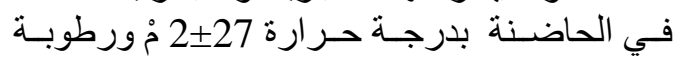

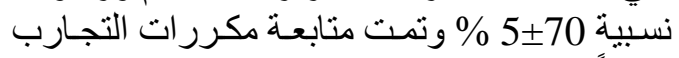

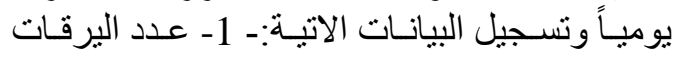

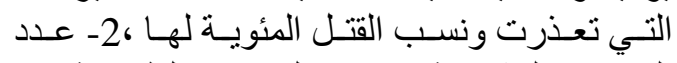

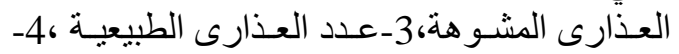

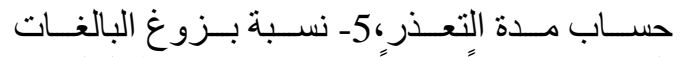

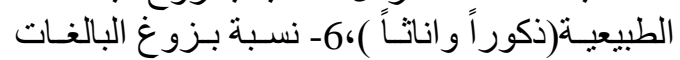

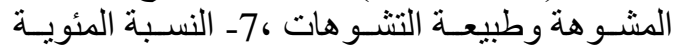

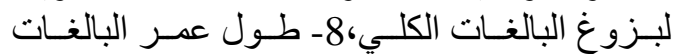

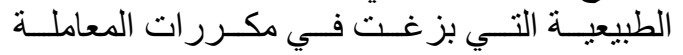
و السبطرة،9- حســاب انتاجيـة البالغـات مـن الانـات

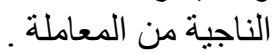

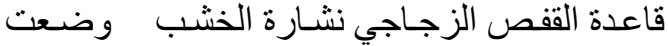

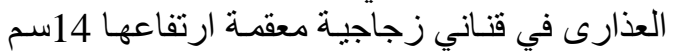

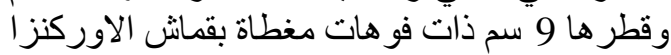

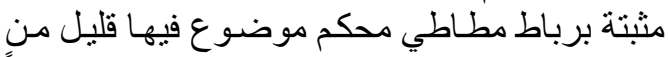

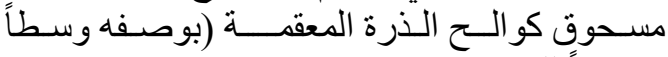

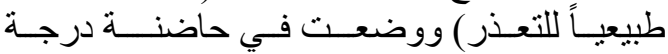

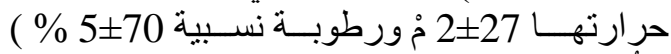

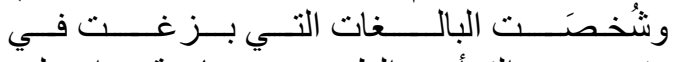

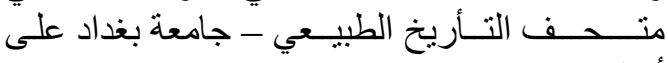
أنها Ceratitis capitata وضعت بالغات الحشرة التي نم الحصول عليها

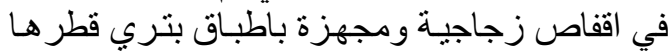

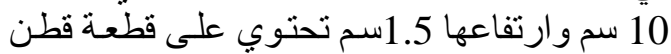

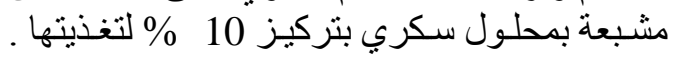

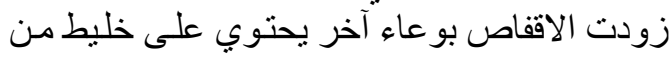

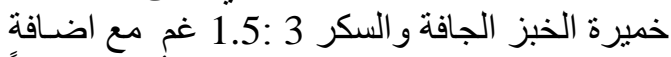

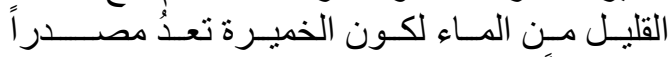

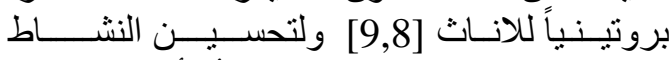

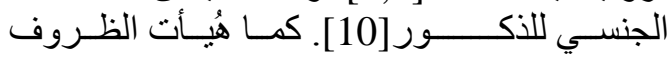

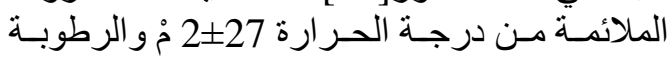

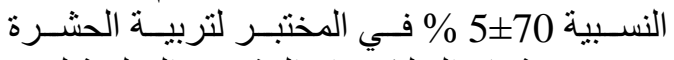

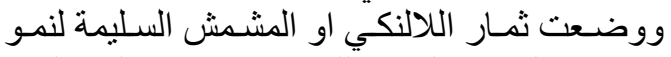

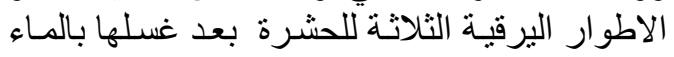

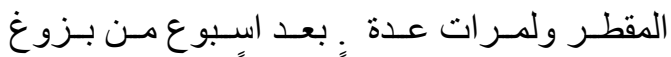

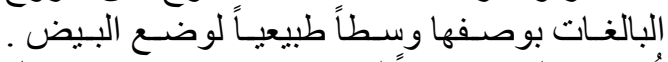

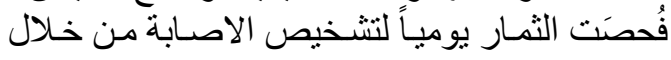

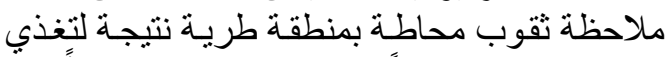

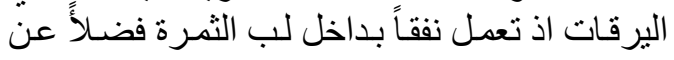

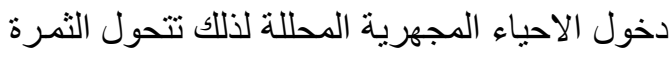

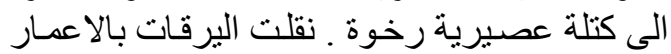

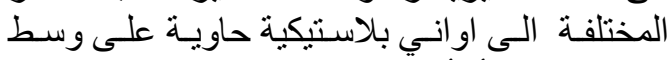

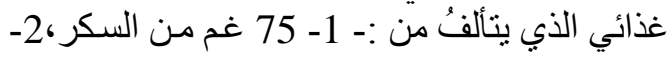

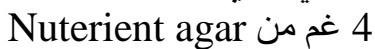

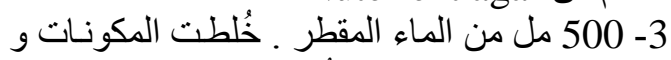

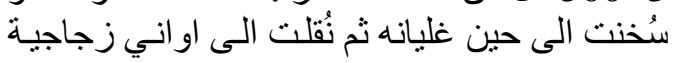

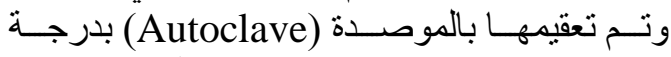
حر ارة 121 مْ وضغنط بالموي 1 جو جو ولمدة 15 دقيقة

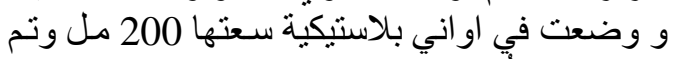

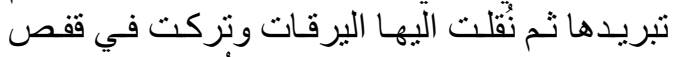

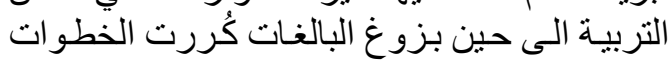

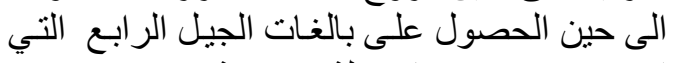

استخدمت بوصفها سلالة مختبرية [11] ل

جمع النبات وتحضير المستخلص الكحولي :-

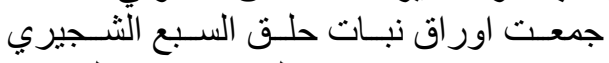

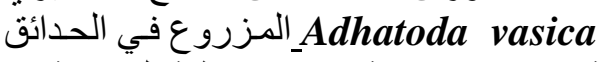
الَعامة في مجمع جامعة بغداد - منطقة الجادرية في الدي

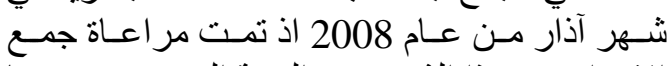

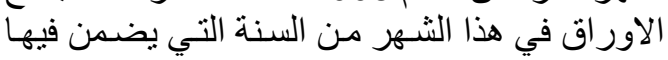

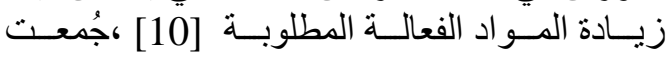


بلغت فيها مدة التعذر 6.06 يوم ، وقد يعود السبب

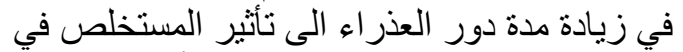

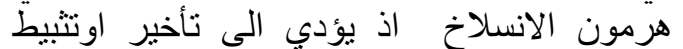

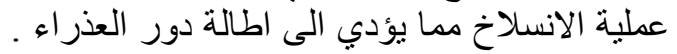

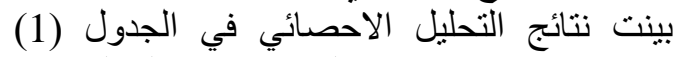

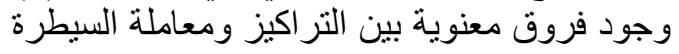

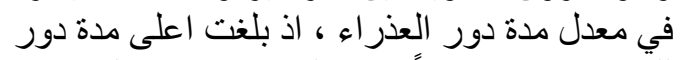

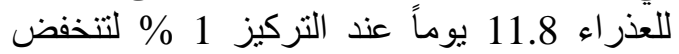
بزيادة الثركيز ، اذ بلغت اقل مدة لدأل الدور العذر اء 3.5

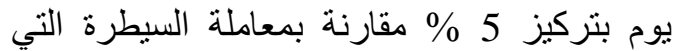

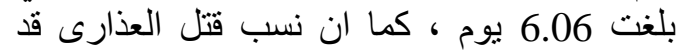

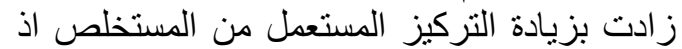

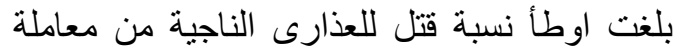
اليرقات بالمستخلص الكحولـي 13.30 \% \% عند التركيز 1 \% لترتفعَ وتصل التى 73.33 \% 33.3 \% عند

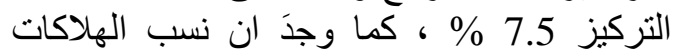

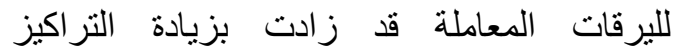

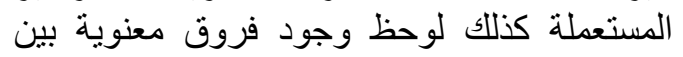

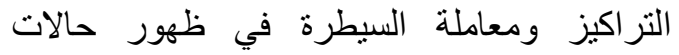

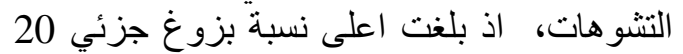

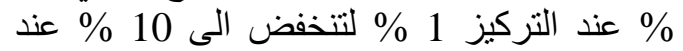
التركيز 5 \% ، اما البالغات المشو هة فبلغت 10 \% \%

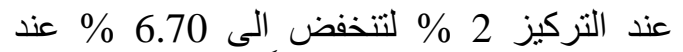

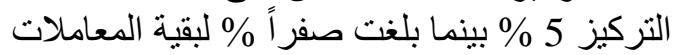

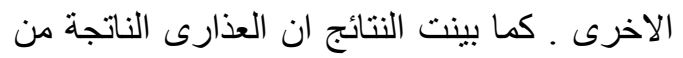

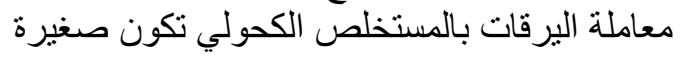

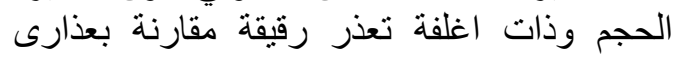

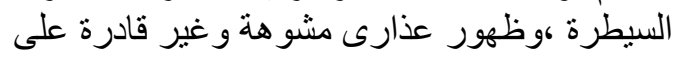

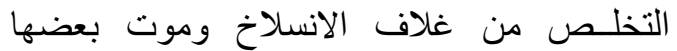

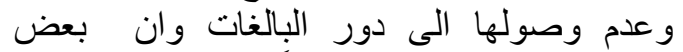

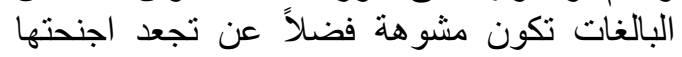

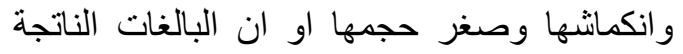

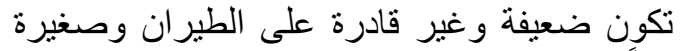
جداً مقارنة بالسيطرة ولم تتمكن من من العيش مدة العيرة

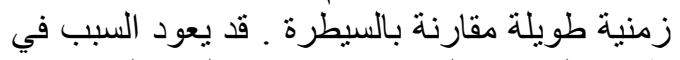

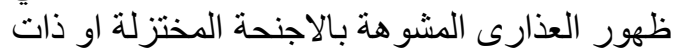

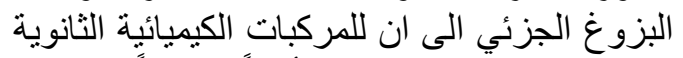

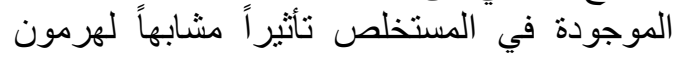

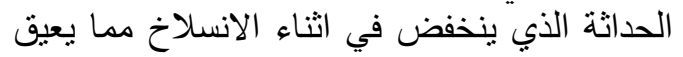

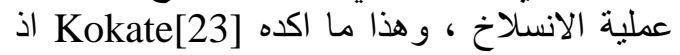
اكد ان الزيوت الطيارة للنبات الظهرت فلئل فعالية

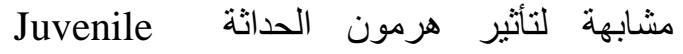
Dusdercus koenigii في حشرة hormone واظهرت الزيوت الطيارة تأثير اً طارداً لحشرتي

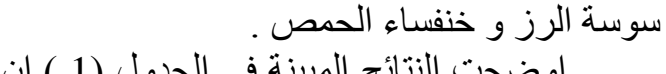

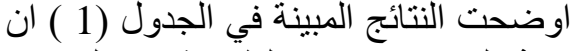
نسب بزوغ الذكور والاناث الطبيعية من العذارى الطئي

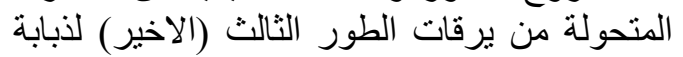
البحر الابيض المنوسط المعاملة بالتر اكيز المقترحة المبنة

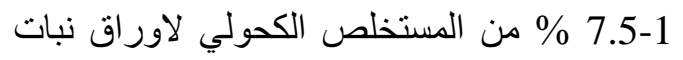

\section{النتائج والمناقشة :}

تبين النتائج الموضحة في الجدول

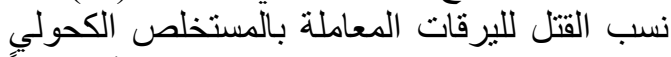

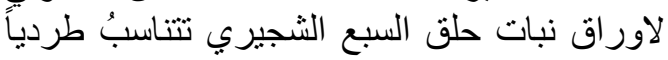

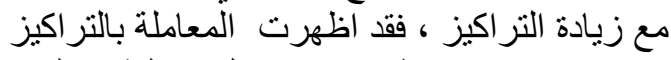

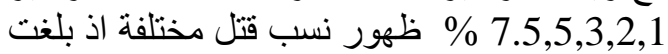
اعلى نسبة قتل لليرقات 66.67 \% 6 \% عند التركيز

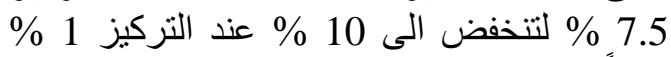

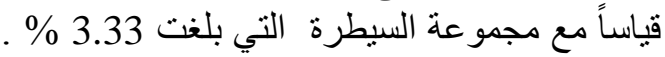
اذ اوضحت محمود [17] ان معاملة برقات النيط الطور

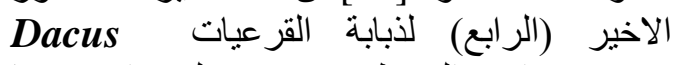
ciliatus بين 10-90 \% اللمستخلص الكحولي الاثيلي لبذور

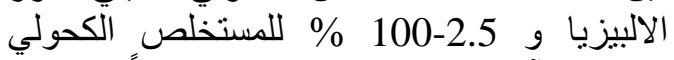

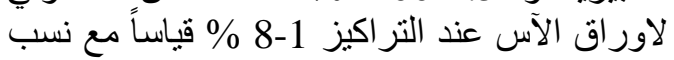

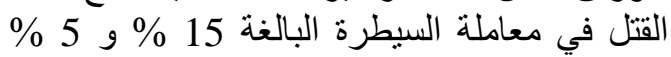

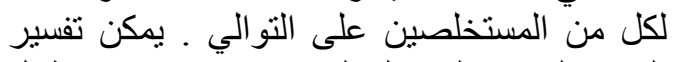

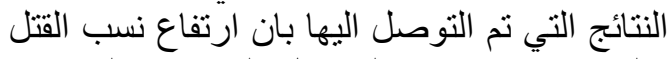

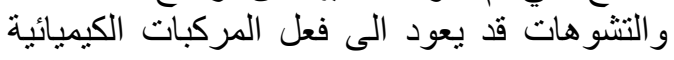

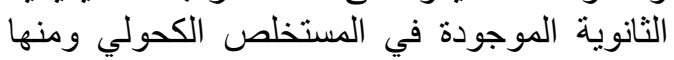

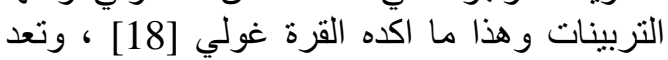

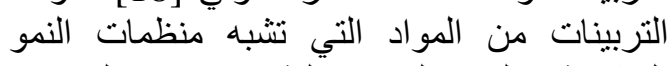

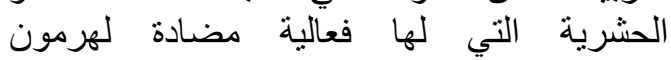
الانسلاخ]20,19] ـ إنّ النتائج التي تم التوصل التهل اليها

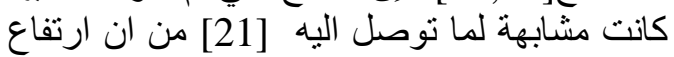

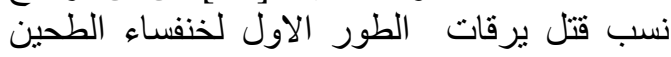
الصدئية Tribolium castaneum الطند اضافة

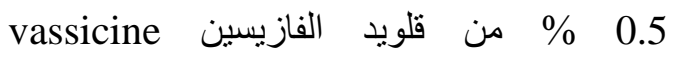

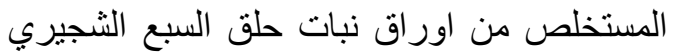
الى الوسط الغذائي ليرقات الطون الطور اليرقي الأون

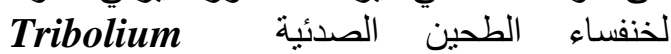
castaneum

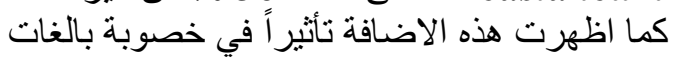

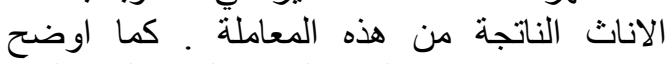
Sadek[22]

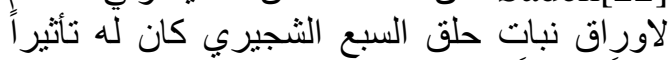

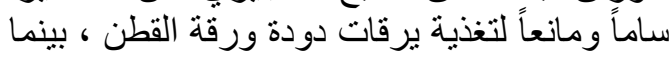

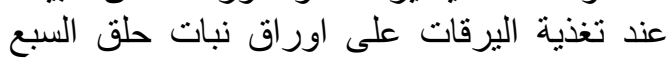

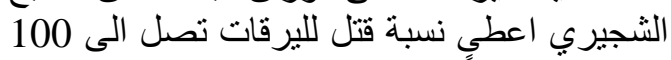
\% بعد 26 يوماً ، و وان هذا المستخلص لإير عالي السمية

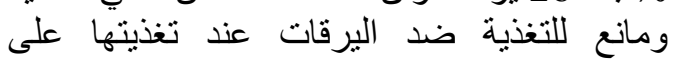

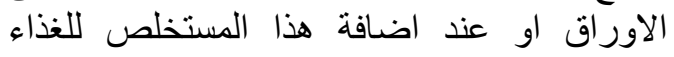

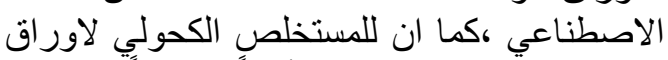

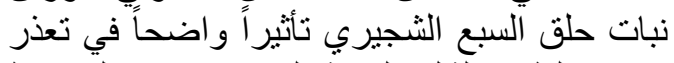

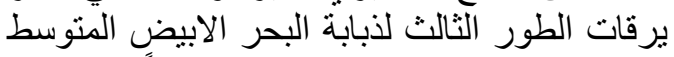

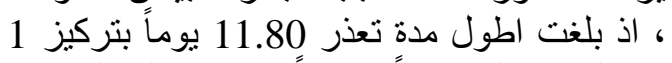
\% التي شكلت فرقاً معنوياً عن معاملة السيطرة

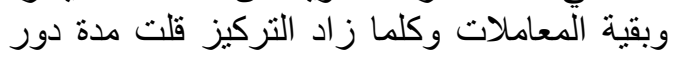

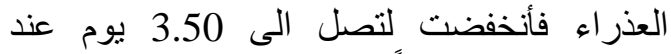
التركيز 5 \% قياساً مع مجموعة السيطرة التي 
مجمو عة السيطرة التي بلغت 93.34 \% ، و واخفقت

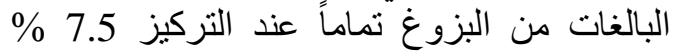

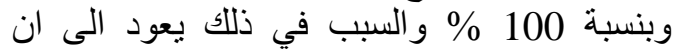
66.67 \% من اليرقات المعاملة قد هلكت داك اما الجزء الى

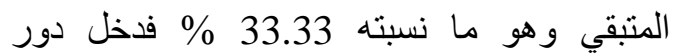
العذر أ الا انها ماتت ولم تصل الى دور البالغات.
حلق السبع الشجيري قد انخفضت بشكل معنوي التئي

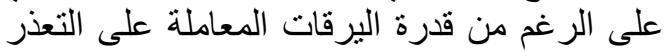

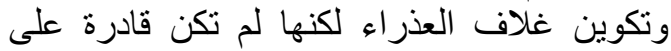

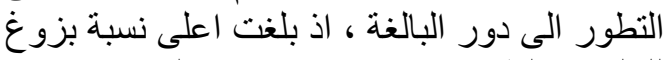

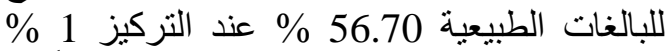
لتنخفض الى 6.66 \% عند التركيز 5 \% \% قياساً مع \%

جدول (1) تأثير تراكيز مختلفة من المستخلص الكحولي لاوراق نبات حلق السبع الثجيري Adhatoda vasica

\begin{tabular}{|c|c|c|c|c|c|c|c|c|c|c|c|c|}
\hline \multicolumn{2}{|c|}{ متوسط عمر البالغات الطبيعية } & \multicolumn{5}{|c|}{ \% بزوغ البالغات د.E } & \multirow{2}{*}{$\begin{array}{l}\text { الغذارى قتل } \\
\text { S.E } \pm \\
\text { S.E }\end{array}$} & \multirow{2}{*}{ 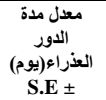 } & \multirow{2}{*}{$\begin{array}{l}\text { \% اليرقتات } \\
\text { S.E } \pm\end{array}$} & \multirow{2}{*}{ 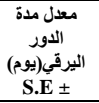 } & \multirow{2}{*}{ 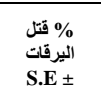 } & \multirow{2}{*}{ التركيز } \\
\hline 우 & $\sigma^{\lambda}$ & جزئي & مشوه & المجموع & & & & & & & & \\
\hline $\begin{array}{c}17.00 \pm 0.14 \\
a\end{array}$ & $\begin{array}{c}18.76 \pm 0.27 \\
\mathrm{a}\end{array}$ & $\begin{array}{c}0.00 \pm 0.00 \\
b\end{array}$ & $\begin{array}{c}0.00 \pm 0.00 \\
b\end{array}$ & $\begin{array}{r}93.34 \pm 0.33 \\
\text { a }\end{array}$ & $\begin{array}{c}46.67 \pm 0.0 \\
a\end{array}$ & $\begin{array}{c}46.67 \pm 0.0 \\
a\end{array}$ & $\begin{array}{c}3.33 \pm 3.33 \\
\text { e }\end{array}$ & $\begin{array}{c}6.06 \pm 0.06 \\
b\end{array}$ & $\begin{array}{c}96.67 \pm 3.33 \\
\mathrm{a}\end{array}$ & $\begin{array}{c}1.06 \pm 0.06 \\
\text { b }\end{array}$ & $\begin{array}{c}3.33 \pm 3.33 \\
e^{*}\end{array}$ & Control \\
\hline $\begin{array}{c}2.00 \pm 0.03 \\
b\end{array}$ & $\begin{array}{c}2.86 \pm 0.29 \\
b\end{array}$ & $\begin{array}{c}20.0 \pm 0.00 \\
\text { a }\end{array}$ & $\begin{array}{c}0.00 \pm 0.00 \\
b\end{array}$ & $\begin{array}{c}56.70 \pm 0.33 \\
\text { b }\end{array}$ & $\begin{array}{c}20 \pm 0.00 \\
\text { b }\end{array}$ & $\begin{array}{c}36.7 \pm 0.00 \\
\text { b }\end{array}$ & $\begin{array}{c}13.30 \pm 3.69 \\
\text { de }\end{array}$ & $\begin{array}{c}11.80 \pm 2.61 \\
\mathrm{a}\end{array}$ & $\begin{array}{c}90.00 \pm 0.00 \\
\text { a }\end{array}$ & $\begin{array}{c}3.47 \pm 0.36 \\
\text { a }\end{array}$ & $\begin{array}{c}10.00 \pm 0.00 \\
\text { e }\end{array}$ & 1 \\
\hline $\begin{array}{c}1.90 \pm 0.10 \\
b\end{array}$ & $\begin{array}{c}2.66 \pm 0.08 \\
b\end{array}$ & $\begin{array}{c}13.3 \pm 0.67 \\
\text { ab }\end{array}$ & $\begin{array}{c}10.0 \pm 0.58 \\
a\end{array}$ & $\begin{array}{c}33.33 \pm 0.33 \\
\text { c }\end{array}$ & $\begin{array}{c}13.33 \pm 0.0 \\
\text { b }\end{array}$ & $\begin{array}{c}20 \pm 0.00 \\
\text { c }\end{array}$ & $\begin{array}{c}23.34 \pm 4.16 \\
\text { d }\end{array}$ & $\begin{array}{c}10.73 \pm 1.88 \\
\text { a }\end{array}$ & $\begin{array}{c}80.00 \pm 0.00 \\
\text { b }\end{array}$ & $\begin{array}{c}2.68 \pm 0.53 \\
a\end{array}$ & $\begin{array}{c}20.00 \pm 0.00 \\
d\end{array}$ & 2 \\
\hline $\begin{array}{c}0.33 \pm 0.33 \\
\text { b }\end{array}$ & $\begin{array}{c}2.00 \pm 1.15 \\
b\end{array}$ & $\begin{array}{c}13.3 \pm 0.67 \\
\text { ab }\end{array}$ & $\begin{array}{c}6.7 \pm 0.33 \\
\text { ab }\end{array}$ & $\begin{array}{c}13.34 \pm 0.33 \\
d\end{array}$ & $\begin{array}{c}3.34 \pm 0.00 \\
\text { bc }\end{array}$ & $\begin{array}{c}10 \pm 0.00 \\
\text { cd }\end{array}$ & $\begin{array}{c}36.66 \pm 4.76 \\
\mathrm{c}\end{array}$ & $\begin{array}{c}4.53 \pm 0.29 \\
b\end{array}$ & $\begin{array}{c}70.00 \pm 0.00 \\
\text { c }\end{array}$ & $\begin{array}{c}1.78 \pm 0.04 \\
\text { b }\end{array}$ & $\begin{array}{c}30.00 \pm 0.00 \\
\text { c }\end{array}$ & 3 \\
\hline $\begin{array}{c}0.33 \pm 0.33 \\
\text { b }\end{array}$ & $\begin{array}{c}0.66 \pm 0.66 \\
b\end{array}$ & $\begin{array}{c}10.0 \pm 0.58 \\
\text { ab }\end{array}$ & $\begin{array}{c}0.00 \pm 0.00 \\
\text { b }\end{array}$ & $\begin{array}{c}6.66 \pm 0.67 \\
\text { de }\end{array}$ & $\begin{array}{c}3.33 \pm 0.00 \\
\text { c }\end{array}$ & $\begin{array}{c}3.33 \pm 0.00 \\
\text { d }\end{array}$ & $\begin{array}{c}33.34 \pm 8.67 \\
\text { b }\end{array}$ & $\begin{array}{c}3.50 \pm 0.76 \\
\text { bc }\end{array}$ & $\begin{array}{c}50.00 \pm 5.77 \\
\text { d }\end{array}$ & $\begin{array}{c}1.58 \pm 0.22 \\
b\end{array}$ & $\begin{array}{c}50.00 \pm 5.77 \\
\text { b }\end{array}$ & 5 \\
\hline$-_{\mathbf{b}}^{* *}$ & $-_{\mathbf{b}}^{* *}$ & $-_{\mathbf{b}}^{* *}$ & $-_{\mathbf{b}}^{* *}$ & $-_{\mathbf{e}}^{* *}$ & $-^{* *}$ & $-^{* *}$ & $\begin{array}{c}33.33 \pm 0.00 \\
a\end{array}$ & $\begin{array}{c}0.00 \pm 0.00 \\
c\end{array}$ & $\begin{array}{c}33.33 \pm 3.33 \\
\text { e }\end{array}$ & $\begin{array}{c}1.43 \pm 0.06 \\
b\end{array}$ & $\begin{array}{c}66.67 \pm 3.33 \\
a\end{array}$ & 7.5 \\
\hline
\end{tabular}

7- Rajani,M. and K. Pundarikakshuda. 1996. Anote on the seasonalvariation of alkaloids in Adhatoda vasica Ness. Intern. J. pharma. 34(4):308-309

8-Drew,R.A.I., $1987 . \quad$ behavioral strategies of fruit flies of the genus Dacus (Diptera:Tephritid) SIGNIFICANT IN MATING \& host-plant realationships. Bull Entomol.Res. 77:73-81

9- Dohary, K.L.1987.Bionomic of fruit flies Daus spp. In some fruits. Ind. J.Entomol. 45(4):406-413.

10-Shroeder,W.J.,D.L. Chambers, and R.Y.Miyabara. 1973. Reproduction of the melon fly, mating activity and mating compatibility of flies treated to function I sterile-release program. J.Econ.Entomol.66:661663.

$$
\begin{aligned}
& \text { 11-الجبوري ، ابر اهيم .2007. ذبابة ثمار فاكهة } \\
& \text { البحر المتوسط افة في بساتين الحمضيات }
\end{aligned}
$$

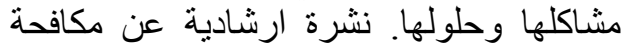

$$
\begin{aligned}
& \text { ذبابة البحر الابيض المتوسط ـ } 43 \text { صفحة الثة عن }
\end{aligned}
$$

12- Harborn, J.B. 1973. Phytochemical methods aguid to modern technique of plant analysis. Halsted press. John wiley \& sons. Newyork. p:278.

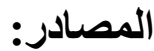

1- Baquar,S.R. 1989 . Medicinal and poisonous of pak . j . Med. 9(186): 95-96.

2--Board, N. 2004. Hand books of herbs cultivation and processing. National Institute of Industrial. pp:400.

3-Dymoch, w.1972.Pharmacographia India. The Institute of health \&Tibbi Researchi. P:50-54.

4- Joshi, B.S.,M.G. Newtown, D.W. Lee, A.D.Barber, and Pelletier.s.w. 1996. Reversal of absolute stereochemeistry of the pyrrolo(2,1b) quinazoline alkaloids vasicine, vasicinone, vasicinol and vasicinolone. J. Science Directtetrahedron. 7(1): 25-28.'

5- Mahmoudian, M.H. Jalilpour and P.Salehian.2002 . Toxicity of Peganum harmala : review and case report. Iran J. pharma . thera $.1(1): 1-4$.

6- Paliwa. J.K., A.K. and Dwived, R.C.Singhs \& Gutpa. 2000. Pharmacokinetics and insito absorption studies of anew antiallergic compound in rats. J.med..20, 197:213-20 
السيسبان في حياتية عثة درنات البطاطا .

$$
\text { رسالة ماجستير / كلية ديالى. }
$$

19- Nakajam ,S. and K. Kawazu .1987 . Heterocycles $10: 117$. In.Hedin ,P. A.(ed) (1983). Plant resistance to insects . Acs . Symposium. Ser.208. Maple Press.p. 93

20-Salama , K . 1983. Plant resistance to insects. AC. Symposium .Ser. 208 Maple Press.p. 92

21- Saxena, B.P., K. Tikku ,C.K. Atal and O. Koul. 1986. Insect antifertility and antifeedant allelochemics in Adhatoda vasica. Insect science and its applications. 7., 4,:489-493.

22- Sadek ,M.M. 2003. Antifeedant and toxic activity of Adhatoda vasica leaf extract against Spodoptera littoralis (Lepidoptera :Noctuidae) . j.appl. entomol. 127. 7 :396-404.

23- Kokate ,C.K., Apte.S.S and Ashok.R. 1985. Anti insect and juvenoidal activity of phytochemical derived from Adhatoda vasica. Ind. J. nat. prod,. $1.2: 7-9$.
13- Harborn,J.B. 1984. Phytochemical methods, aguide to modern technique of analysis. Chapman and Ttall, $2^{\text {nd }}$ ed. Newyork. P:288.

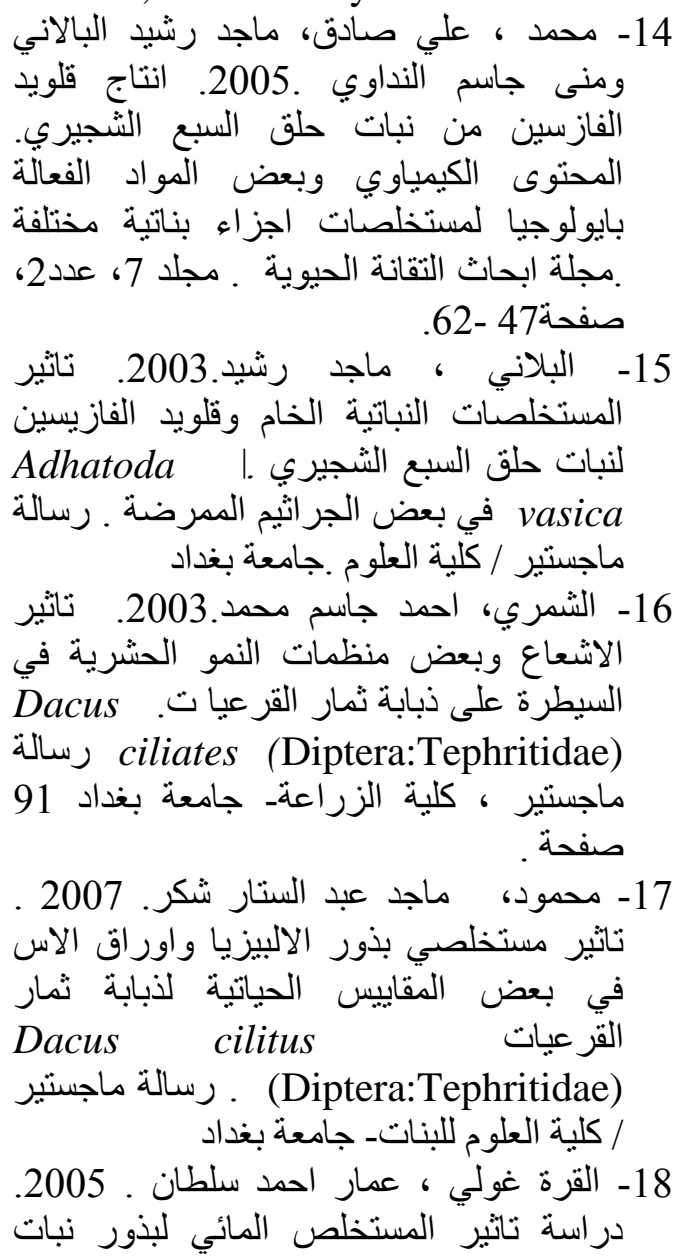




\title{
Effect of alcoholic extract of Adhatoda vasica leaves on some biological performance of Ceratitis capitata larvae
}

\section{Emad A. Mahmood* Ayad A. AL-Taweel** Hiba S. Ahmaed*}

*College of science for womenlUniversity of Baghdad

**Ministry of science and technology /Agriculture research center.

\begin{abstract}
:
Many biological tests were done to study the effect of the crude alcoholic leaves extracts on the biological characteristics at the larvae fruit fly Ceratitis capitata with different concentrations of the extracts exposed 1,2,3,5 and $7.5 \%$ at constant laboratory conditions includes the temperature and the relative humidity $\left(27 \pm 2 C^{0} \& 70 \pm 5 \%\right.$ respectively).

The results of the experiments showed that the alcoholic extracts of the plant effected the growth and the development of the larvae and pupae according to the concentrations. The alcoholic extracts proved to have the highest mortality tend to $66.76 \%$ in larva treated with alcoholic at concentration $7.5 \%$ the result also showed that, there was no signifecant differences on the perid of larva pupation that treated with the different concentration.
\end{abstract}

